Non-newtonian pseudoplastic fluids: Analytical results and exact solutions

M. Guedda, R. Kersner

PII: S0020-7462(11)00062-X

DOI: doi:10.1016/j.ijnonlinmec.2011.04.009

Reference: NLM 1845

To appear in: International Journal of Non-

www.elsevier.com/locate/nlm Linear Mechanics

Received date: 27 October 2009

Revised date: $\quad 7$ March 2011

Accepted date: 5 April 2011

Cite this article as: M. Guedda and R. Kersner, Non-newtonian pseudoplastic fluids: Analytical results and exact solutions, International Journal of Non-Linear Mechanics, doi:10.1016/j.ijnonlinmec.2011.04.009

This is a PDF file of an unedited manuscript that has been accepted for publication. As a service to our customers we are providing this early version of the manuscript. The manuscript will undergo copyediting, typesetting, and review of the resulting galley proof before it is published in its final citable form. Please note that during the production process errors may be discovered which could affect the content, and all legal disclaimers that apply to the journal pertain. 


\title{
Non-Newtonian pseudoplastic fluids: analytical results and exact solutions
}

\author{
M. Guedda ${ }^{a, *}$, R. Kersner ${ }^{b}$ \\ ${ }^{a}$ LAMFA, CNRS UMR 6140, UPJV, Amiens, France \\ ${ }^{b}$ University of Pécs, PMMK, Pécs, Hungary
}

\begin{abstract}
A one layer model of laminar non-Newtonian fluids (Ostwald-de Waele model) past a semi-infinite flat plate is revisited. The stretching and the suction/injection velocities are assumed to be proportional to $x^{\frac{1}{1-2 n}}$ and $x^{-1}$, respectively, where $n$ is the power-law index which is taken in the interval $\left(0, \frac{1}{2}\right)$. It is shown that the boundary-layer equations display both similarity and pseudosimilarity reductions according to a parameter $\gamma$, which can be identified as suction/injection velocity. Interestingly, it is found that there is a unique similarity solution, which is given in a closed form, if and only if $\gamma=0$ (impermeable surface). For $\gamma \neq 0$ (permeable surface) we obtain a unique pseudosimilarity solution for any $0 \neq \gamma \geq-\left(\frac{(n+1)}{3 n(1-2 n)}\right)^{\frac{n}{n+1}}$. Moreover, we explicitly show that any pseudosimilarity solution exhibits similarity behavior and it is, in fact, similarity solution to a modified boundary-layer problem for an impermeable surface. In addition, the exact similarity solution of the original boundary-layer problem is used, via suitable transverse translations, to construct new explicit solutions describing boundary-layer flows induced by permeable surfaces.
\end{abstract}

Keywords. Boundary-layers; Non-Newtonian power-law fluids; Pseudosimilarity; Similarity; Translation groups; Integral equation method.

\section{Introduction and main results}

Many problems in boundary-layer theory lead to particular exact solutions which are characterized by similarity velocity profiles and agree with experimental observations and numerical simulations. It is the purpose of this work to investigate possible conditions for similarity and pseudosimilarity solutions to a class of the boundary-layer flows of laminar non-Newtonian fluids. The range of non-Newtonian fluid behavior exhibited by industrial liquids is very large and the mathematical formulation is, in general, complex. A broad description of the behavior in both steady and unsteady flow situations, together with mathematical models, can be found for example, to mention a few, in Astin et al. [1], Astarita et al. [2], Barnes [3], Bird [4], Tanner [5], Schowalter [6], Rajagopal et al. [7] and Rajacopal [8], [9]. The most frequently used model in non-Newtonian fluid mechanics is the Ostwald-de Waele model, or the non-Newtonian power-law fluid, for which the shear stress $\tau$ is related to the strain rate $u_{y}$ via the expression $[10]-[21]$

$$
\tau=\nu\left|u_{y}\right|^{n-1} u_{y},
$$

where the subscript $y$ denotes the partial derivative with respect to $y, \nu$ is a positive constant and $n>0$ is the power-law index. The case $n<1$ is referred to as pseudo-plastic or shear-thinning fluids, and the case $n>1$ is known as dilatant or shear-thickening fluids. The Newtonian fluid is a special case where the power-law index $n$ is one.

To begin with, we give a brief description of the problem. Consider a steady boundary-layer flow due to a moving plane surface in a quiescent fluid. The fluid can be injected or sucked. For the first

\footnotetext{
*Corresponding author: guedda@u-picardie.fr
} 
approximation, the model is described by the Prandtl equations or the boundary-layer equations for non-Newtonian power-law fluids $[10]-[21]$

$$
\left\{\begin{array}{l}
u_{x}+v_{y}=0 \\
u u_{x}+v u_{y}=\nu\left(\left|u_{y}\right|^{n-1} u_{y}\right)_{y}
\end{array}\right.
$$

with the boundary conditions

$$
\left\{\begin{array}{l}
u(x, 0)=u_{w}(x), \quad v(x, 0)=v_{w}(x) \\
u(x, \infty)=\lim _{y \rightarrow \infty} u(x, y)=0
\end{array}\right.
$$

The Cartesian coordinates $(x, y)$ are such that the $x \geq 0$ coordinate is along the plate and the $y \geq 0$ coordinate is normal to it with $y=0$ is the plate. Subscripts $x$ and $y$ denote partial derivatives with respect to those variables and $u$ and $v$ are the velocity components along the $x-$ and $y$-axes, respectively. The stretching and suction/injection velocities are assumed to be of the form:

$$
u_{w}(x)=U_{0}\left(\frac{x}{l}\right)^{m}, \quad v_{w}(x)=V_{0}\left(\frac{x}{l}\right)^{p}, \quad p=\frac{m(2 n-1)-n}{n+1},
$$

where $n \neq \frac{1}{2}$ and $m=\frac{1}{1-2 n}(p=-1)$. $U_{0}$ and $V_{0}$ are constants and the number $l$ (the characteristic length) is the distance $x$ where the stretching velocity equals $U_{0}$. It is assumed that the positive $x$-direction is that of the main stream, so that $U_{0}>0$. The positive and negative values of $V_{0}$ correspond there to the injection and suction, respectively. The case $V_{0}=0$ means that the plate is impermeable.

In two-dimensional incompressible flow can be characterized by the stream function $\psi$ which is defined in the usual way as $u=\psi_{y}$ and $v=-\psi_{x}$. Thereby, the first partial differential equation (PDE for short) in (2) is satisfied automatically, and the second one reduces to

$$
\psi_{y} \psi_{x y}-\psi_{x} \psi_{y y}=\nu\left(\left|\psi_{y y}\right|^{n-1} \psi_{y y}\right)_{y}
$$

which has to be solved subject to the boundary conditions

$$
\psi_{y}(x, 0)=U_{0}\left(\frac{x}{l}\right)^{\frac{1}{1-2 n}}, \quad \psi_{x}(x, 0)=-V_{0}\left(\frac{x}{l}\right)^{-1}, \quad \psi_{y}(x, \infty)=0
$$

Let us note that if we rewrite (5) as

$$
\psi_{y} \psi_{x y}-\psi_{x} \psi_{y y}=n \nu\left|\psi_{y y}\right|^{n-1} \psi_{y y y}
$$

then we see for $n \neq 1$ that this PDE is degenerate or singular in the sense that the coefficient $\left|\psi_{y y}\right|^{n-1}$ of the highest-order derivative $\psi_{y y y}$ vanishes or blows up at the points where $\psi_{y y}$ vanishes. Hence, equation (5) needs not admit a classical solution. So, one of the important questions is to obtain a family of exact or explicit solutions (similarity solutions, pseudosimilarity solutions, traveling wave,...) which play a fundamental role in finding certain physical and mathematical properties of the general solutions.

A common question in boundary-layer problems is related to solutions having the usual similarity form

$$
\psi(x, y)=x^{\alpha} f(\eta), \quad \eta=y x^{-\beta},
$$

where $\alpha$ and $\beta$ are constants. Research on this subject has a long history, which dates back to the pioneering works by Blasius [22] and Falkner and Skan [23] for the Newtonian case $(n=1)$. The first analysis of the boundary-layer equations for a power-law fluid is due to Schowalter [11] and Acrivos et al. [10] for the case of impermeable walls and to Thomson and Snyder [12], [13] for permeable walls. In the first paper the author derived the equations governing the similarity flow of a pseudoplastic fluid. In [10], using numerics, similarity solutions for both shear-thinning and shear-thickening fluids are obtained. The results of [10] showed that the boundary-layer is of finite-width for $n>1$ (see also [14]). Using a 
phase-plane method similar results are obtained by Pavlov et al. [15]. Physically and mathematically, this phenomenon has been reported in the literature and motivated some renewed interest in analytical and numerical studies (see the papers by Denier and Dabrowski [18], Filipussi et al. [19], Zheng et al. [20], Guedda [21] and the references therein). In all the above papers the number $m$ is assumed to satisfy the condition $1+m(2 n-1)>0$.

The present paper aims to study problem (5), (6), under the condition $1+m(2 n-1)=0$. Our work is motivated by the recent results presented by Magyari et al. [24] for Newtonian fluids and by Guedda [21] for non-Newtonian power-law fluids $\left(n>\frac{1}{2}\right)$. In their interesting paper Magyari et al., using a natural point-mechanical analogy, conjectured several results, most of them were supported by numerical calculations. The authors showed that there is no similarity solution in the usual form (7). To solve the problem, they added the term $\gamma \ln (x)$ to expression (7) which supposes that the surface is permeable. In this way they showed that the boundary-layer equations are reduced to an ordinary differential equation, and obtained for (and only for) $\gamma \geq \gamma^{\star}(=1.079131)$ new solutions called missing solutions or pseudosimilarity solutions (see Section 2 and Section 6). Moreover, the authors showed numerically that for the threshold value $\gamma^{\star}$ the solution is unique but above it multiple solutions are encountered for every given value of $\gamma$.

Although most of similarity and pseudosimilarity solutions were studied by numerical simulations in boundary-layer problems, it seems worthwhile to mathematically discuss some exact solutions, which may allow one to develop or test numerical methods. With this end in view problem (5), (6), where $m=\frac{1}{1-2 n}$ and $n>\frac{1}{2}$, were treated analytically in [21]. It is shown that $\gamma^{\star} \geq\left(\frac{n+1}{3 n(2 n-1)}\right)^{\frac{n}{n+1}}$ for any $n>\frac{1}{2}$ and that the the pseudosimilarity solutions share for $\gamma>\gamma^{\star}$ the remarkable property of multiplicity.

Assuming merely that $0<n<\frac{1}{2}$ the purpose of the present paper is to discus in detail the questions of existence and uniqueness of similarity and pseudosimilarity solutions to problem (5), (6). The essence of our main result is the conclusion that the boundary-layer problem under consideration has a solution in the usual similarity form (7) if and only if the surface is impermeable $(\gamma=0)$, and this solution is expressed in a closed form. For permeable surfaces pseudosimilarity solutions are obtained for all $0 \neq \gamma>-\left(\frac{(n+1)}{3 n(1-2 n)}\right)^{\frac{n}{n+1}}$. In addition, the present boundary-layer problem serves as a motivation to unfold and study mathematical and physical connection between similarity and pseudosimilarity solutions. We show first that the pseudosimilarity reduction is invariant under arbitrary pseudosimilarity variables and, secondly, we demonstrate by an elementary argument that any possible pseudosimilarity solution (for permeable surfaces) is in fact similarity solution in the usual form of a modified boundary-layer problem for an impermeable surface for any $n \neq \frac{1}{2}$, including the Newtonian case. Moreover, using translation invariance groups, new explicit solutions describing boundary-layer flows induced by permeable surfaces are derived for $0<n<\frac{1}{2}$. To the best of our knowledge, these results have not been obtained before.

\section{Reformulation of the problem}

Frequently, in the boundary-layer theory, one looks for general pseudosimilarity solutions of the form

$$
\psi(x, y)=h(x) F(\eta, \zeta)
$$

where $\eta=y \chi(x)$ is the similarity variable and $\zeta=\zeta(x, y)$ is the pseudosimilarity variable which may be taken as independent of $y$. By a pseudosimilarity solution, we mean a solution to (5), (6) having the form $(8)$, such that in the new coordinates $(\eta, \zeta)$ the PDE satisfied by $F$ is particularly simple. In a nutshell, the present discussion will be applied to all $\frac{1}{2} \neq n>0$. This is pedagogically justified and can shed some light on non-similarity techniques.

Our approach is a slight reformulation of the analysis developed 45 years ago for the classical FalknerSkan wedge flows for the Newtonian case [25]. In order not to unnecessarily treat $h$ and $\chi$ as unknown functions we assume that $\chi=B x^{-\beta}$ and $h \equiv A$, where $\beta=\frac{1}{2 n-1}$ and $A$ and $B$ are real parameters such 
that $\nu A^{n-2} B^{2 n-1}=1$ and $A B=U_{0} l^{-m}[21]$; i.e.

$$
A=\nu^{\frac{1}{n+1}}\left(U_{0} l^{1 /(2 n-1)}\right)^{\frac{2 n-1}{n+1}}, \quad B=\nu^{-\frac{1}{n+1}}\left(U_{0} l^{1 /(2 n-1)}\right)^{\frac{2-n}{n+1}} .
$$

In terms of the new variables the boundary-layer equation becomes

$$
\left(\left|F_{\eta \eta}\right|^{n-1} F_{\eta \eta}\right)_{\eta}-m\left(F_{\eta}\right)^{2}=x \frac{d \zeta}{d x}\left[F_{\eta} F_{\zeta \eta}-F_{\zeta} F_{\eta \eta}\right]
$$

where we have assumed that $\zeta=\zeta(x)$. Equation (10) must be a PDE for $F$, so that

$$
x \frac{d \zeta}{d x}=H(\zeta)
$$

where $H \not \equiv 0$ is an arbitrary continuous function. In this way

$$
\left(\left|F_{\eta \eta}\right|^{n-1} F_{\eta \eta}\right)_{\eta}-m\left(F_{\eta}\right)^{2}=H(\zeta)\left[F_{\eta} F_{\zeta \eta}-F_{\zeta} F_{\eta \eta}\right]
$$

The reduction of the pseudosimilarity PDE (12) will be performed with the aid of a modified first level of truncation [26]. The main idea is to neglect only the term $F_{\zeta \eta}$. This is plainly justified for (additive variables separation)

$$
F(\eta, \zeta)=f(\eta)+g(\zeta)
$$

where $f$ and $g$ are arbitrary smooth functions. Inserting (13) into (12) leads to

$$
\left(\left|f^{\prime \prime}\right|^{n-1} f^{\prime \prime}\right)^{\prime}+\beta f^{2}+\gamma f^{\prime \prime}=0
$$

where the primes denote derivatives with respect to the similarity variable $\eta$, and

$$
H(\zeta) \frac{d g}{d \zeta}=-\frac{V_{0}}{A} l \equiv \gamma
$$

In view of the boundary conditions we have

$$
f^{\prime}(0)=1, \quad f^{\prime}(\infty)=0 .
$$

The first surprising property of the above argument is found using the original variable $x$. The function $g$ is expressed as

$$
g(\zeta)=\gamma \ln (x)+\text { constant }
$$

irrespective of the function $H$. Consequently, the stream function has an universal expression:

$$
\psi(x, y)=A(f(\eta)+\gamma \ln (x))+\sigma,
$$

for some number $\sigma$, whenever $f$ exists. This confirms the pseudosimilarity expression obtained by Magyari et al. [24]. The peculiarity of the pseudosimilarity expression (18) is that the condition on $f(0)$ is unnecessary since (14) will be considered as a nonlinear second order differential equation for $\varphi=f^{\prime}$, i.e.

$$
\left(\left|\varphi^{\prime}\right|^{n-1} \varphi^{\prime}\right)^{\prime}+\gamma \varphi^{\prime}+\beta \varphi^{2}=0 .
$$

The boundary conditions read

$$
\varphi(0)=1, \quad \varphi(\infty)=0 .
$$

This is the problem we are going to deal with in the next sections, keeping in mind that if $n>1 / 2$ the problem admits solutions only for large $\gamma$. In passing we note that an initial attempt to solve numerically (19), (20) via the shooting method failed because, as we shall see, solutions of the initial value problem for (19) are not always global. 
Note that the above pseudosimilarity reduction and the nonintuitive expression (17) lead to a wide range of pseudosimilarity variables $\zeta$, and to a family of pseudosimilarity solutions, for $\gamma \neq 0$, which have the property that a stream function can be obtained from another one by a simple translation and has the same shape $\varphi$ or $f$.

Important characteristics of the flow, namely the (non-dimensional) velocity $\mathbf{v}=(u, v, 0)$ and the wall shear stress $\tau_{w}(x)$ are obtained from $\varphi$ as follows:

$$
\begin{gathered}
u(x, y)=u_{w}(x) \varphi(\eta), \quad \eta=\frac{y}{x} R_{x}^{1 /(n+1)}, \\
v(x, y)=\frac{u_{w}(x)}{2 n-1} R e_{x}^{-1 /(n+1)} \eta \varphi(\eta)+\frac{V_{0} l}{x},
\end{gathered}
$$

or, equivalently,

$$
v(x, y)=\frac{u_{w}(x)}{2 n-1} R e_{x}^{-1 /(n+1)} \eta \varphi(\eta)-\frac{\gamma A}{x},
$$

and

$$
\tau_{w}(x)=u_{w}^{2}(x) R e_{x}^{-1 /(n+1)}\left|\varphi^{\prime}(0)\right|^{n-1} \varphi^{\prime}(0) .
$$

So that the value $\varphi^{\prime}(0)$ has to be given with sufficient accuracy. In the above $R e_{x}$ represents the local Reynolds number for non-Newtonian fluids; $R e_{x}=\frac{u_{w}^{2-n}(x) x^{n}}{\nu}$. Note that the number $\gamma$ can be written as $\gamma=-V_{0} U_{0}^{(1-2 n) /(n+1)} l^{n /(n+1)} \nu^{-1 /(n+1)}$. This parameter is usually referred to as the suction/injection parameter. The quantities $\varphi$ and $\varphi^{\prime}(0)$ are named the similar down stream velocity and similar shear stress, respectively. It should be noted here that the Prandtl velocity profile $u / u_{w}$ depends only on the similarity variable $\eta$, which is the essence of similarity reduction reported in a voluminous literature.

Finally, we stress once more that the present pseudosimilarity reduction is invariant under arbitrary pseudosimilarity change of variables. Moreover, as we have just seen, the velocity component $u$ is still unchanged, but the velocity component $v$ deviates from the usual similarity structure. We shall come back to this in the next two sections.

\section{Pseudosimilarity or similarity reduction?}

As mentioned before, problem (19), (20) was considered in [24] for $n=1$ and in [21] for $n>\frac{1}{2}$, where it was found that the existence of solutions had required large positive values of parameter $\gamma$. This shows, in particular, the nonexistence of similarity solutions to the boundary-layer problem in its usual form. A more intuitive way to analyze this circumstance is to suppose that the suction/injection velocity $v_{w}$ is an unknown function. So the aim of this section is twofold: to identify $v_{w} \not \equiv 0$ such that the physical problem may have similarity or pseudosimilarity solutions (a necessary condition) and establish a correspondence between similarity solutions and pseudosimilarity solutions. The starting point is to note that the continuity equation in system (2) is equivalent to

$$
u_{x}+\left(v-v_{w}\right)_{y}=0,
$$

where the suction/injection velocity $v_{w}$ is unknown function to be determined. In terms of the new stream function $\Psi$, according to

$$
u=\Psi_{y}, \quad v-v_{w}=-\Psi_{x},
$$

we obtain the slightly modified PDE

$$
\Psi_{y} \Psi_{x y}-\Psi_{x} \Psi_{y y}=\nu\left(\left|\Psi_{y y}\right|^{n-1} \Psi_{y y}\right)_{y}-v_{w}(x) \Psi_{y y}
$$

accompanied by the following boundary conditions

$$
\Psi_{y}(x, 0)=U_{0}\left(\frac{x}{l}\right)^{\frac{1}{1-2 n}}, \quad \Psi_{x}(x, 0)=\Psi_{y}(x, \infty)=0 .
$$


The only hypothesis in this brief analysis is to assume that the stream function has the usual similarity form; that is

$$
\Psi(x, y)=A x^{q} h(\eta), \quad \eta=B y x^{-r},
$$

where $A$ and $B$ are given by (9). Repeating step by step the reduction argument we deduce that $q=0, r=\frac{1}{2 n-1}$ and that the function $\phi=h^{\prime}$ satisfies

$$
\left\{\begin{array}{l}
\left(\left|\phi^{\prime}\right|^{n-1} \phi^{\prime}\right)^{\prime}+\gamma_{0}(x) \phi^{\prime}+\beta \phi^{2}=0, \\
\phi(0)=1, \quad \phi(\infty)=0
\end{array}\right.
$$

where $\beta=\frac{1}{2 n-1}$ and $\gamma_{0}(x)=-\frac{v_{w}(x) x}{A}$. Therefore, we may conclude that either $\gamma_{0}$ is a constant or a function of $\eta$. Since $\gamma_{0}$ does not contain $y$ there exists a number $\gamma\left(=-\frac{V_{0} l}{A}\right)$ such that

$$
\gamma_{0} \equiv \gamma
$$

which leads to (19) and (20). This line of analysis shows that a similarity reduction to the modified boundary-layer problem is possible only if the suction/injection velocity has the following exact expression

$$
v_{w}(x)=-\frac{A \gamma}{x}=\frac{V_{0}}{x}
$$

which is precisely the suction/injection velocity condition considered in [21] and [24]. Note that $\psi$ is connected to the new stream function $\Psi$ via

$$
\psi(x, y)=\Psi(x, y)+A \gamma \ln (x)
$$

and that the analysis is not explicitly restricted to $n<\frac{1}{2}$.

An interesting question is that what happens if (28) is not satisfied? In this case the parameter $\gamma_{0}$ becomes a function $x ; \gamma_{0}=\gamma_{0}(x)$. If $\gamma_{0}$ varies slowly with distance this quantity may be regarded as a constant parameter. Thus local pseudosimilarity solutions will be obtained by solving problem (27) at different location $x$.

The issue of the pseudosimilarity approach, which has a remarkable degree of simplicity, is clarified by showing that pseudosimilarity solutions are in fact similarity solutions to the modified boundarylayer equations, whenever solutions to (19) and (20) exist, and that the additive variables separation hypothesis (13) emerges naturally from the expression of the new stream function $\Psi$, for any $n \neq \frac{1}{2}$. Those pseudosimilarity solutions can be referred to as similarity solutions of the second kind of the boundary-layer problem (2)-(4). In 1963 Jones and Watson [25, p. 243] mentioned that solutions given by (18) or by a stream function of the general form

$$
\psi(x, y)=h(x) f(\eta)+\psi_{0}(x)
$$

are similarity solutions in the sense that the velocity component $u$ has the same shape across any transverse section of the layer.

Let us return to the modified boundary-layer problem. Clearly, if we put $v_{w} \equiv 0$ problem (25), (26) is exactly the original boundary-layer problem for impermeable surfaces. Note here that the boundary conditions (26) are preserved, except the transpiration velocity distribution which equals now to zero (impermeable surface). Conversely, if $\Psi$ is a solution to the modified PDE (25) the new function $\psi(x, y)=$ $\Psi(x, y)-\psi_{0}(x)$, where $\psi_{0}$ an arbitrary function, satisfies

$$
\psi_{y} \psi_{x y}-\psi_{x} \psi_{y y}=\nu\left(\left|\psi_{y y}\right|^{n-1} \psi_{y y}\right)_{y}+\left(\psi_{0}^{\prime}(x)-v_{w}(x)\right) \psi_{y y}
$$

So that if $\psi_{0}^{\prime}(x)=v_{w}(x)$ the above PDE reduces to (5). In this way the function $\Psi$, which corresponds to the stretching velocity $u_{w}$ for the impermeable surface, generates a solution describing a flow induced by a permeable surface. The stretching velocity $u_{w}$ still invariant, but the boundary layer equation is 
changed. The solution $\Psi$ may be referred to as a primary solution according to the recent work by Magyari [27] (see Section 5).

Let us conclude this section with the following observation. In contrast to the case $n>\frac{1}{2}$, here the number $\beta$ is negative. Consequently, as mentioned before, a local solution with an arbitrary shear stress parameter $\varphi^{\prime}(0)=\omega$ is not necessary global, even for positive values of $\gamma$ (see below).

\section{Similarity solutions for impermeable surfaces}

In this section we are mainly interested in finding similarity solutions for problem (5), (6) in the case of impermeable surfaces. This situation will be nicely illustrated by a simple explicit solution. Since $v_{w} \equiv 0$ $(\gamma=0)$ we are concerned with solutions to

$$
\left\{\begin{array}{l}
\left(\left|\varphi^{\prime}\right|^{n-1} \varphi^{\prime}\right)^{\prime}+\beta \varphi^{2}=0 \\
\varphi(0)=1, \quad \varphi(\infty)=0
\end{array}\right.
$$

To solve the above problem we consider the initial value problem

$$
\left\{\begin{array}{l}
\left(\left|\varphi^{\prime}\right|^{n-1} \varphi^{\prime}\right)^{\prime}+\beta \varphi^{2}=0 \\
\varphi(0)=1, \quad \varphi^{\prime}(0)=\omega
\end{array}\right.
$$

where the number $\omega$ is the shooting (shear stress) parameter. Varying $\omega$ one expects to find a solution which is global and goes to 0 at infinity. Note that if we look for solutions under the form

$$
\varphi_{0}(\eta)=k\left(\eta_{0}-\eta\right)^{-q}
$$

where $k, \eta_{0}$ and $q$ are positive constants, we find that

$$
q=\frac{1+n}{2-n}, \quad k^{n-2}=\frac{|\beta|}{n q^{n}(1+q)} \quad \text { and } \quad \omega=q \eta_{0}^{-q-1}
$$

give rise to blowing-up solution.

Consequently, if $\omega>0$ any solution to (32) is not global. So, the task is to obtain, by an explicit construction, $\omega$ such that the (unique) local solution to (32) is global and satisfies the desired condition at infinity. Note that any local solution satisfies

$$
\left|\varphi^{\prime}(\eta)\right|^{n-1} \varphi^{\prime}(\eta)=|\omega|^{n-1} \omega+|\beta| \int_{0}^{\eta} \varphi^{2}(s) d s,
$$

on $\left(0, \eta_{\omega}\right)$, where $\left(0, \eta_{\omega}\right)$ being the maximal interval of existence. It follows immediately that if $\varphi$ is a global (bounded) solution to (32) the following property

$$
|\omega|^{n-1} \omega=-|\beta| \int_{0}^{\infty} \varphi^{2}(s) d s
$$

holds. Therefore, the shouting parameter has to be negative. On the other hand, the ordinary differential equation in (32) has the first integral $E=$ constant, where the function $E$ is given by

$$
E=\frac{n}{n+1}\left|\varphi^{\prime}\right|^{n+1}+\frac{\beta}{3} \varphi^{3}
$$

We may conclude, therefore, that the global structure of solution curves (in the phase-plane) are simply given by

$$
\varphi^{\prime}= \pm\left[\frac{n+1}{n}\left(c+\frac{|\beta|}{3} \varphi^{3}\right)\right]^{\frac{1}{n+1}}, \quad c=\text { constant }
$$




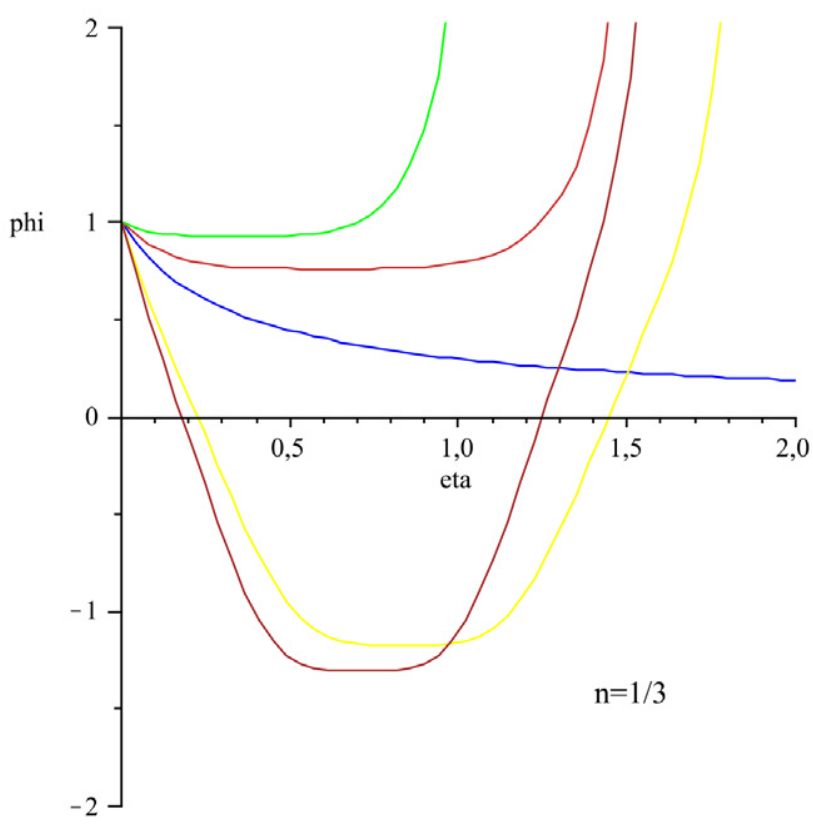

Figure 1: Numerical solutions of the initial value problem (32), with $n=1 / 3$. Profiles showing the effect of the shooting parameter $\omega$.

and then

$$
\varphi^{\prime}=-\left[\frac{n+1}{n} \frac{|\beta|}{3} \varphi^{3}\right]^{\frac{1}{n+1}} .
$$

The necessary condition $c=0$ is obtained from the boundary (physical) condition at infinity; $\varphi(\infty)=0$. This leads in particular to $\omega=\omega_{c}$, where

$$
\omega_{c}=-\left(\frac{(n+1)|\beta|}{3 n}\right)^{\frac{1}{n+1}}
$$

The meaning of the above analysis is that (31) has a unique solution and this solution can be found by a simple integration of (36). More precisely the unique algebraic solution $\varphi$ is given by

$$
\varphi(\eta)=\left[1+\frac{2-n}{n+1}\left(\frac{(n+1)|\beta|}{3 n}\right)^{\frac{1}{n+1}} \eta\right]^{-\frac{n+1}{2-n}}
$$

or

$$
\varphi(\eta)=\left[1+\frac{2-n}{n+1}\left|\omega_{c}\right| \eta\right]^{-\frac{n+1}{2-n}} .
$$

Returning to our original physical problem, we may conclude that if $n<\frac{1}{2}$ and $m=\frac{1}{1-2 n}$ the boundary-layer problem (5), (6) (or (2)-(4)) has exactly one similarity solution for impermeable surfaces which is expressed in term of the stream function

$$
\psi(x, y)=\left(U_{0}^{2 n-1} l \nu\right)^{\frac{1}{n+1}}(3 n)^{\frac{1}{n+1}}\left(\frac{n+1}{1-2 n}\right)^{\frac{n}{n+1}}\left[1+\frac{2-n}{n+1}\left(\frac{n+1}{3 n(1-2 n)}\right)^{\frac{1}{n+1}} \eta\right]^{\frac{1-2 n}{2-n}}, \eta=R e_{x}^{\frac{1}{n+1}} \frac{y}{x}
$$




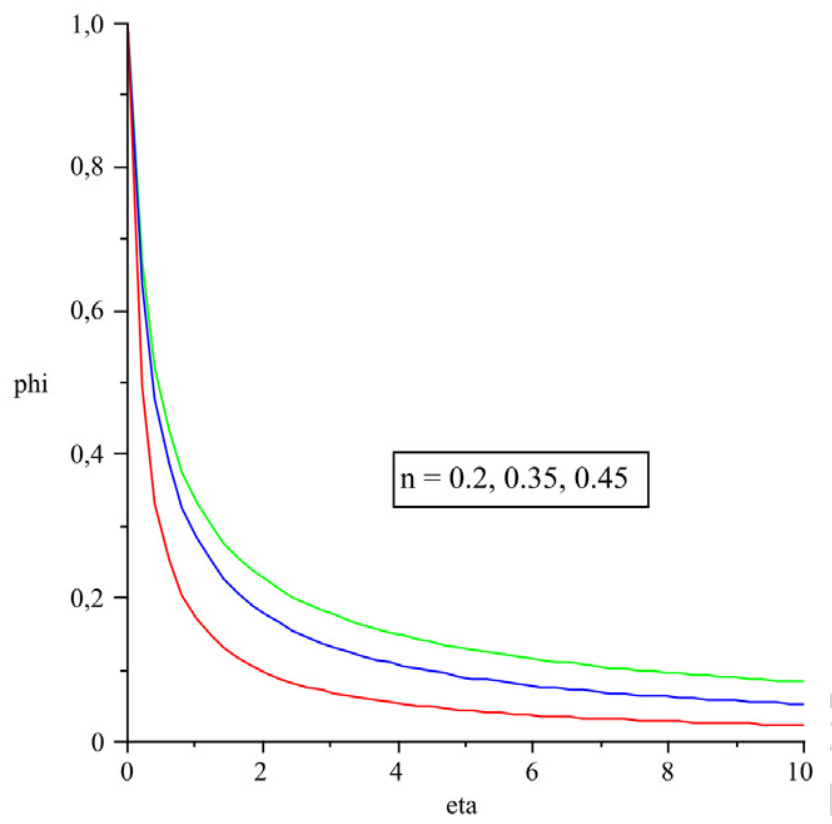

Figure 2: Numerical profiles showing the effect of the power-law index $n$. It is observed that $\varphi(\eta)$ decreases as $n$ increases in the interval [0.2,0.5[. For small values of $n$ the velocity profiles $\varphi(\eta)$ decreases with $n$ (see Figure 3).

The (non-dimensional) velocity $\mathbf{v}=(u, v)$ is obtained as follows

$$
\begin{gathered}
u(x, y)=u_{w}(x)\left[1+\frac{2-n}{n+1}\left(\frac{n+1}{3 n(1-2 n)}\right)^{\frac{1}{n+1}} \eta\right]^{-\frac{n+1}{2-n}}, \\
v(x, y)=\frac{u_{w}(x)}{2 n-1} R e_{x}^{-\frac{1}{n+1}} \eta\left[1+\frac{2-n}{n+1}\left(\frac{n+1}{3 n(1-2 n)}\right)^{\frac{1}{n+1}} \eta\right]^{-\frac{n+1}{2-n}} .
\end{gathered}
$$

The resulting formula for the wall shear stress is

$$
\tau_{w}(x)=-u_{w}^{2}(x) R e_{x}^{-1 /(n+1)}\left(\frac{n+1}{3 n(1-2 n)}\right)^{\frac{n}{n+1}},
$$

which is proportional to $x^{\frac{2 n}{1-2 n}}$. The boundary-layer thickness $\delta(x)$, which is defined as the value of $y$ when $u$ reaches $0.01 u_{w}$, has the explicit form

$$
\delta(x)=\left[10^{\frac{2(2-n)}{n+1}}-1\right]\left[\frac{2-n}{n+1}\left(\frac{n+1}{3 n(1-2 n)}\right)^{-1 /(n+1)}\right] x R e_{x}^{-1 /(n+1)} .
$$

This being derived either from $(40)$ or from the equation $\varphi\left(\eta_{\delta}\right)=0.01$, where $\eta_{\delta}=R e_{x}^{\frac{1}{n+1}} \frac{\delta(x)}{x}$. The boundary-layer thickness is therefore a decreasing function in proportion to $x^{\frac{1}{2 n-1}}$.

Figures 2 and 3 illustrate the power-law index effect. It can be noted that the profile $\varphi(\eta), \eta>0$, tends to 0 as $n$ increases to $1 / 2$, or decreases to 0 . This result can be seen from (39) and from the variation of 


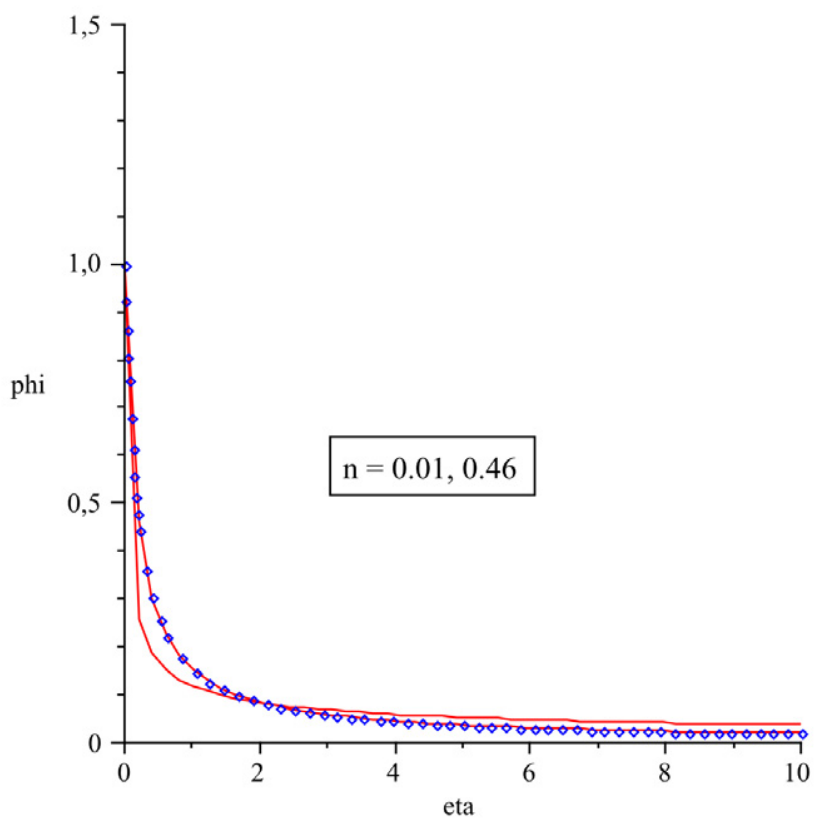

Figure 3: Numerical solutions (full lines) to problem (31) for $n=0.01$ and $n=0.46$. Numerical result is found to be in a full agreement with the exact solution (dotted line).

shear stress parameter $\omega_{c}=-\left(\frac{n+1}{3 n(1-2 n)}\right)^{1 /(n+1)}$ vs $n$. There exists $n_{c}(\approx 0.2552)$ such that $\omega_{c}$ increases for $\left(0, n_{c}\right)$ and decreases for $\left(n_{c}, 1 / 2\right)$. Moreover, $\omega_{c}$ tends to $-\infty$ as $n$ approaches 0 and $1 / 2$.

\section{The translation invariance}

In this section we consider, when $0<n<\frac{1}{2}$, certain exact solutions describing boundary-layer flows induced by permeable surfaces. The key is the invariance property or the translation groups (TG) introduced very recently by Magyari for the Newtonian case [27]. It is proposed here to extend the TG method to non-Newtonian power-law flows. We briefly describe this approach. The idea is based on the fact that equation (5) is invariant under smooth translation

$$
y \rightarrow y+y_{0}(x)
$$

of the transverse coordinate $y$. By this we mean that, thanks to the special structure of the left-hand-side of (5), the function

$$
\widetilde{\psi}(x, y) \equiv \psi\left(x, y+y_{0}(x)\right)
$$

is a solution of equation (5) if it is satisfied by $\psi$. In this way the velocity components $u$ and $v$ are transformed into

$$
\widetilde{u}(x, y)=u\left(x, y+y_{0}(x)\right), \quad \widetilde{v}(x, y)=v\left(x, y+y_{0}(x)\right)-y_{0}^{\prime}(x) \widetilde{u}(x, y),
$$

where $y_{0}^{\prime}=\frac{d}{d x} y_{0}$. Thereby the wall conditions read

$$
\widetilde{u}(x, 0) \equiv \widetilde{u}_{w}(x)=u\left(x, y_{0}(x)\right), \quad \widetilde{v}(x, 0) \equiv \widetilde{v}_{w}(x)=v\left(x, y_{0}(x)\right)-y_{0}^{\prime}(x) \widetilde{u}_{w}(x) .
$$


The TG transformation will not change the boundary condition at infinity. A remarkable consequence of this approach is that a solution to the boundary-layer flow induced by an impermeable surface (primary solution) generates a set of solutions induced by permeable surfaces. Conversely, a primary solution $(u, v)$ can be obtained from a given solution $(\widetilde{u}, \widetilde{v})$ by the displacement $\widetilde{y}_{0}$, which is obtained by solving the following ODE

$$
\widetilde{y}_{0}^{\prime}(x) \widetilde{u}\left(x, \widetilde{y}_{0}(x)\right)-\widetilde{v}\left(x, \widetilde{y}_{0}(x)\right)=0 .
$$

Note that the above ODE admits a trivial solution $\widetilde{y}_{0} \equiv 0$ if and only if $(\widetilde{u}, \widetilde{v})$ is a primary solution.

To illustrate the use of the Magyari approach, we return to problem (5), (6), with $V_{0}=0$. From (40) and (41) we find that the transformed counterpart of the primary velocity components are

$$
\left.\begin{array}{rl}
\widetilde{u}(x, y)= & u_{w}(x)\left[1+\frac{2-n}{n+1}\left(\frac{n+1}{3 n(1-2 n)}\right)^{\frac{1}{n+1}}\left(\eta+\eta_{0}(x)\right)\right]^{-\frac{n+1}{2-n}}, \\
\widetilde{v}(x, y)= & \frac{u_{w}(x)}{2 n-1} \operatorname{Re}_{x}^{-\frac{1}{n+1}}\left(\eta+\eta_{0}(x)\right)\left[1+\frac{2-n}{n+1}\left(\frac{n+1}{3 n(1-2 n)}\right)^{\frac{1}{n+1}}\left(\eta+\eta_{0}(x)\right)\right]^{-\frac{n+1}{2-n}} \\
& -y_{0}^{\prime}(x) \widetilde{u}(x, y),
\end{array}\right\}
$$

where

$$
\eta_{0}(x)=\operatorname{Re}_{x}^{\frac{1}{n+1}} \frac{y_{0}(x)}{x}=R e^{\frac{1}{n+1}} x^{\frac{1}{1-2 n}} y_{0}(x)
$$

is the displacement of the similarity variable $\eta$ corresponding to the displacement $y_{0}(x)$. In (49) Re stands for the Reynolds number for non-Newtonian fluids. The transformed wall conditions are

$$
\left.\begin{array}{rl}
\widetilde{u}_{w}(x)= & u_{w}(x)\left[1+\frac{2-n}{n+1}\left(\frac{n+1}{3 n(1-2 n)}\right)^{\frac{1}{n+1}} \eta_{0}(x)\right]^{-\frac{n+1}{2-n}}, \\
\widetilde{v}_{w}(x)= & \frac{u_{w}(x)}{2 n-1} R_{x}^{-\frac{1}{n+1}} \eta_{0}(x)\left[1+\frac{2-n}{n+1}\left(\frac{n+1}{3 n(1-2 n)}\right)^{\frac{1}{n+1}} \eta_{0}(x)\right]^{-\frac{n+1}{2-n}} \\
& -y_{0}^{\prime}(x) \widetilde{u}_{w}(x)
\end{array}\right\}
$$

or

$$
\widetilde{v}_{w}(x)=\frac{u_{w}(x) x^{-1}}{2 n-1}\left[y_{0}(x)-(2 n-1) x y_{0}^{\prime}(x)\right]\left[1+\frac{2-n}{n+1}\left(\frac{n+1}{3 n(1-2 n)}\right)^{\frac{1}{n+1}} \eta_{0}(x)\right]^{-\frac{n+1}{2-n}} .
$$

Here, we shall deal with with two particular cases: $\eta_{0}=$ constant and $y_{0}=$ constant. These cases follow from the general solution of the following ODE

$$
(2 n-1) x y_{0}^{\prime}-y_{0}(x)=-\kappa=\text { constant }
$$

Solving this ODE we find

where $\kappa_{0}$ is a constant, and then

$$
y_{0}(x)=\kappa+\kappa_{0} x^{-\frac{1}{1-2 n}}
$$

$$
\eta_{0}(x)=R e^{\frac{1}{n+1}}\left[\kappa x^{\frac{1}{1-2 n}}+\kappa_{0}\right]
$$

From relation (50) we then see for $\eta_{0}=$ constant $(\kappa=0)$ that the transformed velocity $\widetilde{u}_{w}(x)$ is proportional to $x^{\frac{1}{1-2 n}}$ and the transformed transpiration velocity $\widetilde{v}_{w}=0$. In such situation, it is known that the velocity field is solved in a closed form in a full agreement with (48) (see Section 4). The transformed similar down stream velocity and the transformed boundary-layer thickness are given by

$$
\widetilde{\varphi}(\eta)=\frac{\varphi\left(\eta+\eta_{0}\right)}{\varphi\left(\eta_{0}\right)}, \quad \widetilde{\delta}(x)=\left[1+\eta_{0} \mathcal{N}\right] \delta(x)
$$


where $\mathcal{N}=\frac{2-n}{n+1}\left(\frac{n+1}{3 n(1-2 n)}\right)^{\frac{1}{n+1}}$.

For the case $y_{0}(x) \equiv \kappa$, we have $\eta_{0}(x)=\kappa R e^{\frac{1}{n+1}} x^{\frac{1}{1-2 n}}$. The transformed solution is

$$
\left.\begin{array}{l}
\widetilde{u}(x, y)=u_{w}(x)\left[1+\frac{2-n}{n+1}\left(\frac{n+1}{3 n(1-2 n)}\right)^{\frac{1}{n+1}}\left(\eta+\eta_{0}(x)\right)\right]^{-\frac{n+1}{2-n}}, \\
\widetilde{v}(x, y)=\kappa \frac{u_{w}(x) x^{-1}}{2 n-1}\left[1+\frac{2-n}{n+1}\left(\frac{n+1}{3 n(1-2 n)}\right)^{\frac{1}{n+1}}\left(\eta+\eta_{0}(x)\right)\right]^{-\frac{n+1}{2-n}},
\end{array}\right\}
$$

and corresponds to the wall functions

$$
\begin{gathered}
\widetilde{u}_{w}(x)=u_{w}(x)\left[1+\frac{2-n}{n+1}\left(\frac{n+1}{3 n(1-2 n)}\right)^{\frac{1}{n+1}} \eta_{0}(x)\right]^{-\frac{n+1}{2-n}} \\
\widetilde{v}_{w}(x)=\kappa \frac{u_{w}(x) x^{-1}}{2 n-1}\left[1+\frac{2-n}{n+1}\left(\frac{n+1}{3 n(1-2 n)}\right)^{\frac{1}{n+1}} \eta_{0}(x)\right]^{-\frac{n+1}{2-n}}
\end{gathered}
$$

On the basis of equation (56), the transformed stretching velocity $\widetilde{u}_{w}$ approaches the initial one $u_{w}$ for small values of $x$, but deviates it far downstream. Note that $\widetilde{v}_{w}(x) \sim x^{\frac{2 n}{1-2 n}}$ as $x$ approaches 0 , and that the similarity structure of the solution is broken.

It is worthwhile to observe that we obtain similar conclusions if both $\eta_{0}$ and $y_{0}$ vary with $x$ in accordance with (52) and (53).

Clearly, as mentioned in [27], the TG approach opens new possibilities for constructing several solutions with a regime prescribed on the wall. Those solutions are generated from a given solution by displacement $y_{0}=y_{0}(x)$. Consequently, if the displacement of the transverse coordinate $y_{0}$, or the displacement of the similarity variable $\eta_{0}$ is known, expressions (48), (50) give the velocity filed of the boundary-layer problem corresponding to the stretching and transpiration velocities $\left(\widetilde{u}_{w}, \widetilde{v}_{w}\right)$ given by $(50)$. Conversely, for a given $\left(\widetilde{u}_{w}, \widetilde{v}_{w}\right)$ the TG approach allows us to determine the range of admissible transverse coordinates associated to our primary solution via $(50)$, to construct the solution $(\widetilde{u}, \widetilde{v})$ from $(48)$, and to find the features that distinguish the primary velocity $(u, v)$ from $(\widetilde{u}, \widetilde{v})$, as precisely as possible. Then, under what conditions on $\widetilde{u}_{w} \neq 0$ and $\widetilde{v}_{w} y_{0}$ exists? An immediate answer is formulated in the following compatibility condition

$$
(2 n-1) x \chi^{\prime}-\chi=-(2 n-1) \frac{x \widetilde{v}_{w}}{\widetilde{u}_{w}},
$$

where the function $\chi\left(=y_{0}\right)$ is given by

$$
\chi(x)=M^{-1} x^{\frac{1}{2 n-1}}\left[\left(\frac{\widetilde{u}_{w}}{u_{w}}\right)^{\frac{n-2}{n+1}}-1\right]
$$

with

$$
M=\frac{2-n}{n+1}\left(\frac{n+1}{3 n(1-2 n)} R_{e}\right)^{\frac{1}{n+1}} .
$$

Note that if $\widetilde{u}_{w}=C u_{w}$, for some constant $C$, we deduce from the compatibility condition, as has been shown above, that $\widetilde{v}_{w}=0$.

In the main context of this paper we are interested particularly in finding a solution $(\widetilde{u}, \widetilde{v})$, from the primary solution, which corresponds to the stretching and suction/injection velocities $\widetilde{u}_{w}, \widetilde{v}_{w}$ being proportional to $x^{\frac{1}{1-2 n}}$ and $x^{-1}$ respectively. Clearly, in this circumstance the compatibility condition is violated. In [27], it is indicated that for Newtonian flows the case of inverse linear stretching velocity requires a special approach. 


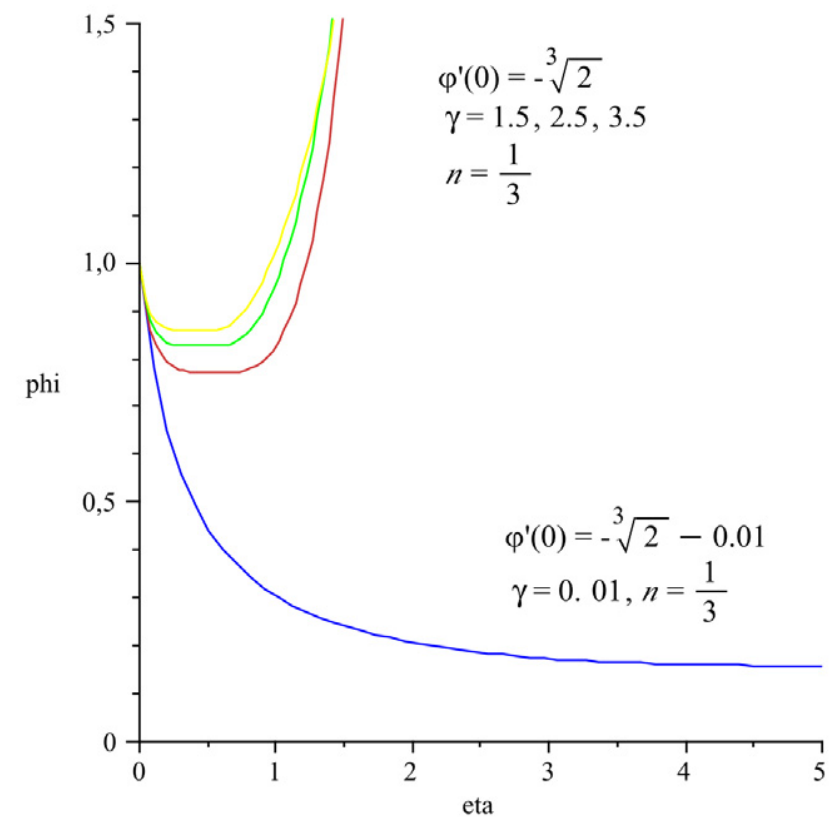

Figure 4: Profiles showing the effect of the parameter $\gamma$.

\section{Pseudosimilarity solutions}

For the post part we will be concerned with a detailed analysis of the solutions for the modified boundary layer problem (25), (26). Hence the problem of interest is the eigenvalue problem (19), (20); that is (for the reader convenience)

$$
\begin{gathered}
\left(\left|\varphi^{\prime}\right|^{n-1} \varphi^{\prime}\right)^{\prime}+\gamma \varphi^{\prime}+\beta \varphi^{2}=0, \quad \eta>0, \\
\varphi(0)=1, \quad \varphi(\infty)=0,
\end{gathered}
$$

where $\beta=-\frac{1}{1-2 n}, 0<n<1 / 2$ and $\gamma \neq 0$. In the light of the analysis of the previous section with $\gamma=0$, it seems natural to expect that problem (60), (61) admits unique solutions for $\gamma \neq 0$ (at least for small $|\gamma|)$. A mathematical question is to provide a range of $\gamma$-the spectrum- for which problem (60), (61) has a solution.

To solve the above problem we use the Crocco variables approach with the help of the theory of the integral equation developed by Gilding and Kersner [28]. Firstly, it is easily checked, by noting that $\beta$ is negative and using the function $E$ (see (34)), that any solution to (60), (61) is monotonic (strictly) decreasing. We introduce a new variable $\theta$ by

$$
\theta(s)=-\varphi^{\prime}(\eta), \quad s=\varphi,
$$

which satisfies the first order degenerate ODE

$$
n \theta^{n} \theta^{\prime}=\gamma \theta-\beta s^{2}, \quad 0<s<1,
$$

or the following integral equation

$$
\theta^{n}(s)=\gamma s+|\beta| \int_{0}^{s} \frac{r^{2} d r}{\theta(r)},
$$


obtained by a simple integration. Thus, the study of solutions to $(60),(61)$ is reduced to that of solutions to equation (64) supplemented by the initial condition

$$
\theta(0)=0
$$

\subsection{Eigensolutions for positive $\gamma$}

It may be noted that the integral equation (64) can be used to obtain the explicit solution to (19), (20) for $\gamma=0$. So, when looking for solutions under the form $\theta_{0}(s)=a s^{\chi}$, it can be shown $(\gamma=0)$

$$
\chi=\frac{3}{n+1}, \quad a=\left(|\beta| \frac{n+1}{3 n}\right)^{\frac{1}{n+1}} .
$$

Hence

$$
\theta_{0}(s)=\left(|\beta| \frac{n+1}{3 n}\right)^{\frac{1}{n+1}} s^{\frac{3}{n+1}} .
$$

In view of (62), the ODE in (31) holds which leads to the explicit solution (38). Consequently, for any $\gamma>0$ problem (65), (64) has a positive solution $\theta_{\gamma}$ defined on $(0,1)[28]$. Moreover, one sees from (63)

$$
\theta_{\gamma}(s) \geq \gamma^{\frac{1}{n}} s^{\frac{1}{n}} \text { and } \theta_{\gamma}(s) \geq \theta_{0}(s)
$$

for all $0 \leq s \leq 1$. Together with the integral equation we deduce

$$
\theta_{\gamma}^{n}(s) \leq \gamma s+|\beta| \int_{0}^{s} \frac{r^{2} d r}{\theta_{0}(r)} \equiv \gamma s+\theta_{0}^{n}(s)
$$

and then

$$
\max \left\{\gamma s,\left(|\beta| \frac{n+1}{3 n}\right)^{\frac{n}{n+1}} s^{\frac{3 n}{n+1}}\right\} \leq \theta_{\gamma}^{n}(s) \leq \gamma s+\left(|\beta| \frac{n+1}{3 n}\right)^{\frac{n}{n+1}} s^{\frac{3 n}{n+1}}
$$

The above estimates show that $\theta_{\gamma}$ tends to $\theta_{0}$ as $\gamma \rightarrow 0$. Moreover

$$
\theta_{\gamma}(s) \sim\left(|\beta| \frac{n+1}{3 n}\right)^{\frac{1}{n+1}} s^{\frac{3}{n+1}}
$$

as $s \rightarrow 0$. Next, we shall refine estimate (70). We assume that $\theta_{\gamma}$ can be written as

$$
\theta_{\gamma}(s)=s^{\frac{3}{n+1}}\left(a+b s^{k}+\ldots\right)
$$

where the constants $b, k$ are to be determined and $a=\left(|\beta| \frac{n+1}{3 n}\right)^{\frac{1}{n+1}}$ (see (70)). Substituting (71) into (63) confirms that

$$
k=\frac{1-2 n}{n+1}, \quad b=\frac{\gamma}{n}\left(|\beta| \frac{n+1}{3 n}\right)^{\frac{1-n}{n+1}} .
$$

Thus

$$
\theta_{\gamma}(s) \sim\left(|\beta| \frac{n+1}{3 n}\right)^{\frac{1}{n+1}} s^{\frac{3}{n+1}}+\frac{\gamma}{n}\left(|\beta| \frac{n+1}{3 n}\right)^{\frac{1-n}{n+1}} s^{\frac{2(2-n)}{n+1}}
$$

as $s \rightarrow 0$.

Returning to problem (60), (61), we deduce that, for any $\gamma \geq 0$ and $0<n<\frac{1}{2}$, all solutions $\varphi$ decay algebraically according to

$$
\varphi(\eta) \sim\left[\frac{2-n}{n+1}\left(\frac{(n+1)|\beta|}{3 n}\right)^{\frac{1}{n+1}} \eta\right]^{-\frac{n+1}{2-n}},
$$


as $\eta$ goes to infinity, irrespective of $\gamma$, showing that the pseudosimilarity solutions behave like the unique similarity solution obtained in Section 4 . Note that estimate (69) can also be used to exhibit an estimate of the shear stress $\varphi^{\prime}(0)$. Clearly, we infer from (69)

$$
-\left[\gamma+\left(|\beta| \frac{n+1}{3 n}\right)^{\frac{n}{n+1}}\right]^{1 / n} \leq \varphi^{\prime}(0) \leq-\max \left\{\gamma^{1 / n},\left(|\beta| \frac{n+1}{3}\right)^{\frac{1}{n+1}}\right\} .
$$

The next analysis deals with the uniqueness result. Let $\gamma>0$ be fixed and $\theta_{\gamma}^{1}$ and $\theta_{\gamma}^{2}$ satisfy (65), (64). Assume that $\theta_{\gamma}^{1}>\theta_{\gamma}^{2}$ on some $\left[0, s_{\gamma}\right], 0<s_{\gamma}<1$. One subtracts the integral equations for $\theta_{\gamma}^{1}$ and $\theta_{\gamma}^{2}$, one gets

$$
\left(\theta_{\gamma}^{1}\right)^{n}(s)-\left(\theta_{\gamma}^{2}\right)^{n}(s)=|\beta| \int_{0}^{s} \frac{r^{2}\left(\theta_{\gamma}^{2}(r)-\theta_{\gamma}^{1}(r)\right)}{\theta_{\gamma}^{1}(r) \theta_{\gamma}^{2}(r)} d r
$$

for all $0 \leq s \leq s_{\gamma}$. The LHS of the latter is positive for $0 \leq s \leq s_{\gamma}$, while the RHS is negative. A contradiction. Thus $\theta_{\gamma}^{1} \equiv \theta_{\gamma}^{2}$ on $\left[0, s_{\gamma}\right]$. Hence, there cannot be two different solutions satisfying (65), (64). Consequently, for any $\gamma \geq 0$ problem (60), (61) has a unique solution for $n<\frac{1}{2}$, and there exists a unique $\omega_{c}=\omega_{c}(\gamma)$ such that $\varphi^{\prime}(0)=\omega_{c}$. In addition, the estimate

$$
\varphi(\eta) \leq\left[1+\frac{2-n}{n+1}\left(\frac{(n+1)|\beta|}{3 n}\right)^{\frac{1}{n+1}} \eta\right]^{-\frac{n+1}{2-n}}
$$

holds in $(0, \infty)$, for all $\gamma \geq 0$. In particular, this indicates that the solution with $\gamma=0$ gives a limiting form for any solution with $\gamma$, and that the profile $\varphi(\eta)$ tends to 0 as the power-law index approaches 0 and $1 / 2$. The $\gamma$ effect is visible in the following estimate

$$
\varphi(\eta) \leq\left[1+\frac{1-n}{n} \gamma^{1 / n} \eta\right]^{-\frac{n}{1-n}},
$$

which shows that $\varphi(\eta)$ decreases as $\gamma$ increases and goes to 0 as $\gamma$ tends to infinity (strong suction velocity). Another property obtained from (77) is the following estimate of the boundary-layer thickness:

$$
\delta(x) \leq \frac{n}{1-n}\left[10^{\frac{2(1-n)}{n}}-1\right] \gamma^{-1 / n} x R e_{x}^{-1 /(n+1)}, \quad \gamma>0 .
$$

The next $\gamma$-effect is found from the large $\gamma$-behavior of the shear stress parameter. Using (69) one sees that $\left|\omega_{c}\right|$ increases with $\gamma$ as

as $\gamma$ approaches infinity.

$$
\omega_{c} \sim-\gamma^{1 / n}
$$

Note also that the shear stress is connected to $\gamma$ via the equation

$$
\left|\varphi^{\prime}(0)\right|^{n-1} \varphi^{\prime}(0)=-\gamma+\beta \int_{0}^{\infty} \varphi^{2}(\eta) d \eta
$$

This indicates, in particular, that the integral term becomes negligible compared to $\gamma$. A similar estimate (for $\varphi^{\prime}(0)$ ) and conclusion are given in [24] for the Newtonian case.

Lastly, we point out, as mentioned before, that our results do differ in one essential way from the earlier works by Magyari et al. [24] for the Newtonian case and by Guedda [21] for $n>\frac{1}{2}$. It is shown that problem (19), (20), white $n>\frac{1}{2}$, has a one parameter family of solutions if (and only if) the suction parameter $\gamma>\gamma^{\star}$; one solution for each specified $\varphi^{\prime}(0)$. More precisely, for any $\gamma>\gamma^{\star}$ there exist a smallest negative initial shear stress $\varphi_{0, \min }^{\prime}(\gamma)$ and a maximal positive initial shear stress $\varphi_{0, \max }^{\prime}(\gamma)$, such that the multiple solutions are parametrized by $\varphi^{\prime}(0)$ in the interval

$$
\varphi_{0, \min }^{\prime}(\gamma) \leq \varphi^{\prime}(0) \leq \varphi_{0, \max }^{\prime}(\gamma)
$$

For $\gamma=2$ it is found that $\varphi_{0, \min }^{\prime}=-1.725126$ and $\varphi_{0, \max }^{\prime}=+9.212868$ [24]. The solution of (19), (20) is only unique for $\gamma=\gamma^{\star}$. On the other hand, all these solutions decay algebraically according to $\gamma(2 n-1) \eta^{-1}$, in contrast to $(73)$, as $\eta \rightarrow \infty$, for $\gamma>\gamma^{\star}$. This result has been proved numerically in [24] for the Newtonian case (see Figure 5 or Figure 3 of [24]). 


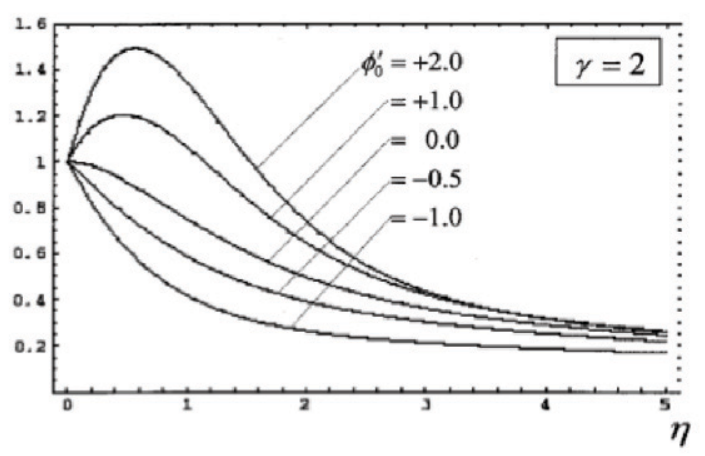

Figure 5: Plots of $\varphi$ versus $\eta$ when $n=1$ and $\gamma=2$, according to Ref [24]. This shows multiple solutions corresponding to different values of $\varphi^{\prime}(0)\left(=\phi_{0}^{\prime}\right)$.

\subsection{Eigensolutions for negative $\gamma$}

We close our analysis with the case $\gamma<0$. We can ask, naturally, if the above results still true if $\gamma$ is negative. The uniqueness result still holds. As for the existence of solutions we note that problem (65), (64) may be viewed as a particular case of the problem

$$
\left\{\begin{aligned}
|\theta(s)|^{n-1} \theta(s) & =\gamma s+|\beta| \int_{0}^{s} \frac{r^{2} d r}{\theta(r)}, \quad 0<s<1 \\
\theta(0) & =0
\end{aligned}\right.
$$

which can be written as

$$
\left\{\begin{aligned}
n|\theta|^{n-1} \theta \theta^{\prime} & =\gamma \theta+|\beta| s^{2}, \\
\theta(0) & =0 .
\end{aligned}\right.
$$

To prevent any ambiguity, we shall consider only positive solutions. If $\gamma=0$, there are two explicit solutions

$$
\theta_{ \pm}= \pm \theta_{0}
$$

where $\theta_{0}$ is given by (66). So problem (80) may have both positive and negative solutions for small $|\gamma|$. To obtain positive solutions, we look for an approximate solution in the polynomial form

$$
\theta_{a p}(s)=s^{\chi}\left(a+b s^{k}+\ldots\right),
$$

where $w, k, a, b$ are some some appropriate constants. By substituting (82) into (81) it is found that

$$
\chi=\frac{3}{n+1}, a= \pm\left(|\beta| \frac{n+1}{3 n}\right)^{\frac{1}{n+1}}, k=\frac{1-2 n}{n+1}, b=\frac{\gamma}{n}\left(|\beta| \frac{n+1}{3 n}\right)^{\frac{1-n}{n+1}} .
$$

Since the approximate solution must to be positive, the number $a$ is given by $a=\left(|\beta| \frac{n+1}{3 n}\right)^{\frac{1}{n+1}}$. From this we may conclude that for sufficiently small $\varepsilon>0$, problem (81) (or (80)) has a positive solution $\bar{\theta}_{\gamma}$ in $(0, \varepsilon)$. This solution is unique and satisfies (72) for small $s$ and

$$
\theta_{0}^{n}(s)+\gamma s<\bar{\theta}_{\gamma}^{n}(s)<\theta_{0}^{n}(s),
$$

as long as $\bar{\theta}_{\gamma}$ is positive. Below we will show that $\bar{\theta}_{\gamma}$ is the positive solution to (65), (64) under a favorable condition on $\gamma<0$. Define

$$
\gamma_{n}^{\star}=\left(\frac{|\beta|(n+1)}{3 n}\right)^{\frac{n}{n+1}}
$$


The task remaining is to show that $\bar{\theta}_{\gamma}$ still positive on $(0,1)$ for

$$
|\gamma| \leq \gamma_{n}^{\star}
$$

Let us suppose that there exists $\varepsilon \leq s_{0}<1$ such that $\bar{\theta}_{\gamma}$ is positive on $\left(0, s_{0}\right)$ and vanishes at $s=s_{0}$. Setting $s=s_{0}$ in (83) we arrive at the inequality

$$
\left(\frac{|\beta|(n+1)}{3 n}\right)^{\frac{n}{n+1}} s_{0}^{\frac{3 n}{n+1}}<|\gamma| s_{0} .
$$

Since $s_{0}<1$ and $n<\frac{1}{2}$, we deduce $|\gamma|>\left|\gamma_{n}^{\star}\right|$, which contradicts (85). Hence $\bar{\theta}_{\gamma}$ is the unique positive solution to (65), (64). Consequently, for any $\gamma \geq-\gamma_{n}^{\star}$ problem (65), (64) has a unique positive solution.

\section{Summary and Conclusions}

We have investigated the boundary-layer behavior of a non-Newtonian pseudoplastic fluid over a continuous (permeable or impermeable) plane surface. The stretching velocity is given by $u_{w}(x)=U_{0}(x / l)^{m}$, where $m=\frac{1}{1-2 n}, 0<n<\frac{1}{2}$, and the suction/injection velocity satisfies $v_{w}(x)=V_{0} l / x$. The analysis reveals that there is a transparent difference between the case $n<\frac{1}{2}$ and the case $n>\frac{1}{2}$. For $n<\frac{1}{2}$ the boundary-layer problem is solved in a closed similarity for (and only for) impermeable surfaces. In term of the stream function, this solution is given by

$$
\psi_{0}(x, y)=\left(U_{0}^{2 n-1} l \nu\right)^{\frac{1}{n+1}}(3 n)^{\frac{1}{n+1}}\left(\frac{n+1}{1-2 n}\right)^{\frac{n}{n+1}}\left[1+\frac{2-n}{n+1}\left(\frac{n+1}{3 n(1-2 n)}\right)^{\frac{1}{n+1}} \eta\right]^{\frac{1-2 n}{2-n}}, \quad \eta=R e_{x}^{\frac{1}{n+1}} \frac{y}{x} .
$$

The second part of the analysis deals with pseudosimilarity solutions for permeable surfaces. In this situation it is shown that the boundary-layer problem in its usual form has no similarity solutions. A modified boundary-layer equation is proposed and pseudosimilarity solutions are presented for any $V_{0} \neq 0$ such that $-V_{0} l / A=\gamma \geq-\gamma_{n}^{\star}=-\left(\frac{(n+1)}{3 n(1-2 n)}\right)^{\frac{n}{n+1}}$. Moreover, we have demonstrated, in particular, that those pseudosimilarity solutions are in fact similarity solutions to a modified boundary-layer problem. In addition, it is found that the stream function behaves like $\psi(x, y) \sim \psi_{0}(x, y)$ for large $\operatorname{Re}_{x}^{\frac{1}{n+1}} \frac{y}{x}$, irrespective of $\gamma>0$. The effect of the suction velocity $\gamma$ is also investigated. It is found analytically that the stream velocity decreases as $\gamma$ increases. The asymptotic behavior of the wall shear stress parameter $\varphi^{\prime}(0)$ at large $\gamma$ is analyzed. It is established that $\varphi^{\prime}(0) \sim-\gamma^{1 / n}$, showing that the wall shear stress $\tau_{w}(x)$ behaves like $\tau_{w}(x) \sim-u_{w}^{2}(x) R e_{x}^{-1 /(n+1)} \gamma$ as $\gamma \rightarrow \infty$. The above similarity stream function $\psi_{0}(x, y)$ is used to find new explicit solutions describing boundary-layer flows induced by permeable surfaces by adapting the TG approach of Magyari [27].

The existence or the nonexistence of positive solutions for $\gamma<-\gamma_{n}^{\star}$ is an open question. However, it is reasonable to expect that the solutions exist for all negative values of $\gamma$. Our investigation may be hampered by the lack of numerical solutions for the integral equation. However, we believe that the results presented here will be used for testing numerical schemata.

Acknowledgement. The authors thank the referee for the careful reading of the original manuscript and for making constructive suggestions, which have improved the presentation of this paper. E. Magyari kindly supplied us with a copy of [27]. The results of Section 5 are based on his ideas. The present work was partially done during a visit of the authors to the LaMUSE, University of Saint-Etienne. The authors wish to thank M. Boukrouche and L. Paoli for the invitation and their kind hospitality. Supported in part by Direction des Affaires Internationales, UPJV, Amiens France. To the memory of Najia Ait Bella.

\section{References}

[1] J. Astin, R.S. Jones, P. Lockyer, Boundary layers in non-Newtonian fluids, J. de Méc. 12 (1973), 525-539. 
[2] G. Astarita, G. Marrucci, Principles of non-Newtonian Fluid Mechanics, McGraw-Hill, 1974.

[3] H.A. Barnes, A brief history of the yield stress, Appl. Rhoel. 9 (6) (1998), 53-78.

[4] R.B. Bird, Non-Newtonian behavior of polymeric liquids, Physica A: Statistical and theoretical Physics 118 (1-3) (1988), 3-16.

[5] R.I. Tanner, Engineering Rheology, Calderon Press, Oxford 1985.

[6] W.R. Schowalter, Mechanics of Non-Newtonian Fluids, Pergamon, Oxford, 1978.

[7] K.R. Rajagopal, A.S. Gupta, A.S. Winemann, On a boundary layer theory for non-Newtonian fluids, Int. J. Eng. Sci. 18 (1980), 875-883.

[8] K.R. Rajagopal, A note on unsteady unidirectional flows of a non-Newtonian fluid, Internat. J. Non-Linear Mech. 17 (1982), no 5-6, 369-373.

[9] K.R. Rajacopal, Mechanics of non-Newtonian fluids, Recent developments in Theoretical Fluid Mechanics (Pskey, 1992) (G.P. Gladi and J. Necas, eds.) Pitman Res. Notes Math. ser., vol. 291, Longman Scientrific \& Technical, Harlow, 1993, pp. 129-162.

[10] A. Acrivos, M.J. Shah, E.E. Peterson, Momentum and heat transfer in laminar boundary flow of non-Newtonian fluids past external surfaces, AIChEJ. 6 (1960) 312-317.

[11] W. R. Schowalter, The application of boundary-layer theory to power-law pseudoplastic fluids: Similar solutions, AIChEJ 6 (1960) 24-28.

[12] E. R. Thomson, W. T. Snyder, Drag reduction of a non-Newtonian fluid by fluid injection at the wall, J. Hydronautics 2 (1968) 177-180.

[13] E. R. Thomson, W. T. Snyder, Laminar boundary-layer flows of Newtonian fluids with nonNewtonian fluid injections, J. Hydronautics 3 (1970) 86-91.

[14] I. Teipel, Discontinuities in boundary-layer problems of power-law fluids, Mech. Res. Commun (1974) 269-274.

[15] K. B. Pavlov, I. A. Fedotov, A.P. Shakhorin, Structure of laminar boundary-layer in non-Newtonian dilatant fluids, Izv. Akad. Nauk SSSR Mekh. Zhid. Gaza 4 (1981) 142.

[16] D. Mansutti, K.R. Rajagopal, Flow of a shear thinning fluid between intersecting planes, Int. J. Non-Linear Mech. (1991), 769-775.

[17] M. Akçay, M.A. Yükselen, Drag reduction of a nonNewtonian fluid by fluid injection on a moving wall, Arch. Appl. Mech. 69 (1999) 215-225.

[18] J.P. Denier, P. P. Dabrowski, On the boundary-layer equations for power-law fluids, Proc. R. Soc. Lond. A 460 (2004) 3143-3158.

[19] D. Filipussi, J. Gratton, F. Minotti, The self-similar laminar boundary-layer of power law nonNewtonian fluids, Nuovo Cim. 116 (2001) 393-402.

[20] L. Zheng, X. Zhang, J. He, Existence and estimate of positive solutions to nonlinear singular boundary value problem in the theory of dilatant non-Newtonian fluids, Mathematical and Computer Modeling Vol. 45 (3-4) (2007) 387-393.

[21] M. Guedda, Similarity and pseudosimilarity solutions of degenerate boundary-layer equations, HandBook of Differential Equations, Stationary partial differential equations, M. Chipot ed., Vol 4. 117198, 2007. 
[22] H. Blasius, Grenzchichten in Flussigkeiten mit kleiner Reibung, Z. Math. Phys. 56 (1908) 1-37.

[23] V.M. Falkner, S.W. Skan, Solutions of the boundary layer equations, Phil. Mag. 12 (1931) 865-896.

[24] E. Magyari, I. Pop, B. Keller, The "missing" similarity boundary-layer flow over a moving plane surface, Z. angew.Math.Phys. 53 (2002) 782-793.

[25] C.W. Jones, E.J. Watson, Two dimensional boundary layers, in : Laminar Boundary Layers, L. Rosenhead ed., Dover Pub., New York, 1963, Chapt. V, Sect. 21.

[26] M. Massoudi, Local non-similarity solutions for the flow of a non-newtonian fluid over a wedge, Int. J. Non-Linear Mech. 36 (2001) 961-976.

[27] E. Magyari, Translation groups of the boundary-layer flows induced by continuous surfaces, J. Fluid Mech. 655 (2010), 327-343.

[28] B. H. Gilding, R. Kersner, Travelling waves in nonlinear diffusion-convection-reaction, Progress in Nonlin. Diff. Equ. Appl. Vol. 60, Birkhauser Verlag, 2004. 
$>$ In this study we examine a class of non-Newtonian pseudoplastic fluids. $>$ The power-law index is taken in the interval $(0,1 / 2)$. $>$ Exact similarity solutions are obtained in a closed form. $>$ New explicit solutions are also obtained using the translation invariance method. 